\title{
THE LUTEINIZING HORMONE-RELEASING HORMONE (LHRH) NEURON SYSTEM IN THE RAT CENTRAL NERVOUS SYSTEM. AN IMMUNOHISTOCHEMICAL STUDY
}

\author{
YASUHIKO IBATA ${ }^{1}$, HITOSHI OKAMURA ${ }^{1}$, KENJI FUKUI ${ }^{2}$, HIROKO L. OBATA- \\ TSUTO $^{1}$, MINORU TANAKA ${ }^{1}$, HIROSHI KIMURA ${ }^{3}$, ETSURO HASHIMURA ${ }^{4}$, SADA- \\ HITO SIN $^{4}$ and KENICHI IMAGAWA ${ }^{4}$ \\ ${ }^{1}$ Department of Anatomy and ${ }^{2}$ Department of Psychiatry, Kyoto Prefectural University of Medicine, \\ Kawaramachi-Hirokoji, Kyoto 602, ${ }^{3}$ Department of Anatomy, Shiga University of Medical Science, Seta, \\ Ohtsu, and 4Otsuka Assay Institute, Otsuka, Pharmaceutical Co., Ltd., Tokushima 770, Japan
}

\begin{abstract}
The luteinizing hormone-releasing hormone (LHRH) neuron system in the rat was investigated by an improved peroxidase-antiperoxidase (PAP) method. LHRH neuronal somata were found in the preoptic area, diagonal bundle of Broca, and surrounding areas under physiological conditions. The LHRH immunoreactive fibers and terminals were widely distributed in the telencephalon, diencephalon, and midbrain. The features of their pathways projecting to several parts, including their main terminal areas in the median eminence and the organum vasculosum of the lamina terminalis, are discussed in comparison with results obtained in other mammalian species, particularly the guinea pig. The possibility is suggested that LHRH is released into the cerebrospinal fluid via the processes of the LHRH neurons intimately associated with the wall of the third ventricle and the aquaductus cerebri.
\end{abstract}

The LHRH neuron system in the mammalian central nervous system (CNS) has been studied by immunohistochemistry in several species $(2-5$, $7-13,17,19-23,26)$. However, it is extremely difficult to locate the neuronal somata of this system in the rat under physiological conditions, in comparison with other mammalian species (2). Conflicting accounts of their location have been reported by different investigators $(1,5,14$, $15,22-24,28)$. We have previously concluded that neuronal somata are located in the ventral part of the medial preoptic area (11). The conclusion was drawn by examining changes in nerve endings in the median eminence (ME) after placing lesions in several parts of the hypothalamus; the lesions were placed because we had failed to detect LHRH immunoreactive neuronal somata by immunohistochemical methods (11). By improving the procedures of immunohistochemistry, however, we later discovered immunoreactive neuronal somata in the rat under completely physiological conditions. Most recently, Bennett-Clarke and Joseph (5) have also succeeded in detecting LHRH neuronal somata in the rat. This paper deals with the location of LHRH neuronal somata and the distribution of their fibers and terminals in the hypothalamus and other regions of the brain.

\section{MATERIALS AND METHODS}

Six adult male Wister rats weighing about $250 \mathrm{~g}$ and housed under a $14 \mathrm{~h}$ light-10 h dark schedule were used. They were perfused via the left cardiac ventricle with a mixture of $4 \%$ paraformaldehyde, $0.2 \%$ picric acid and $0.35 \%$ glutaraldehyde adjusted to $\mathrm{pH} 7.4$ with $0.1 \mathrm{M}$ phosphate buffer. The brain was removed after perfusion and further fixed with the same mixture, but without glutaraldehyde, for $48 \mathrm{~h}$ at $4^{\circ} \mathrm{C}$. Thereafter serial frontal frozen sections (20 $\mu \mathrm{m}$ in thickness) from four brains and serial 
sagittal frozen sections from two brains were stored in phosphate-buffered saline (PBS), solution containing $0.3 \%$ Triton $\mathrm{X}-100$ for 4 days at $4^{\circ} \mathrm{C}$. For immunohistochemistry, sections were first incubated in bovine serum albumin (diluted 100 -fold) for $1 \mathrm{~h}$ at $25^{\circ} \mathrm{C}$, and then incubated in anti-LHRH serum (diluted 8,000fold) for $48 \mathrm{~h}$ at $4^{\circ} \mathrm{C}$. The anti-LHRH serum used in this study was the same as described in the previous investigation (10). Sections were then incubated with anti-rabbit $\operatorname{IgG}$ (diluted 200 -fold) for $2 \mathrm{~h}$ at $25^{\circ} \mathrm{C}$, followed by incubation with peroxidase-antiperoxidase (PAP) (diluted 200 -fold) for $90 \mathrm{~min}$ at $25^{\circ} \mathrm{C}$. Finally, they were exposed for $10 \mathrm{~min}$ at $25^{\circ} \mathrm{C}$ to $3,3^{\prime}$-diaminobenzidine substrate solution containing $\mathrm{H}_{2} \mathrm{O}_{2}$, mounted on glass slides, and examined by light microscopy. Between each step, sections were washed with PBS. Control sections were treated with anti-LHRH serum absorbed with synthetic LHRH. No positive immunoreactive materials were detected in the control sections.

\section{RESULTS}

\section{Location of LHRH Neuronal Somata}

The somata of LHRH neurons were weaker in their immunoreactivity than their processes and terminals. They had a faint brown coloration. In the most rostral area, some of the somata were located in the lateral part of the diagonal bundle of Broca. More caudally, they were at the level of the organum vasculosum lamina terminalis (OVLT). Significant numbers were found in the surrounding area of the OVLT, particularly the dorsolateral portion, and a large numbers of LHRH terminals were also observed in the OVLT (Fig, 1a). These were medium sized bipolar or multipolar neurons. Immunoreactive somata were also scattered in the basal part of the septum. At a more caudal level, a fairly large number of immunoreactive neurons of multiple shapes were located in the laterobasal part of the preoptic area (POA), which is considered to be the lateral part of the pars suprachiasmatica of the POA (preoptic region of suprachiasmatic area, POSA) (Fig. 1b). The processes extending from these somata seemed to be thick and tortuous in the vicinity of their perikarya. At the level of the rostral part of the anterior hypothalamic nucleus, immunoreactive neurons were found located more laterally than those at the POA level (Fig. 1c). They showed the same morphological features as those found in the POSA. At a level caudal to the region where the periventric-
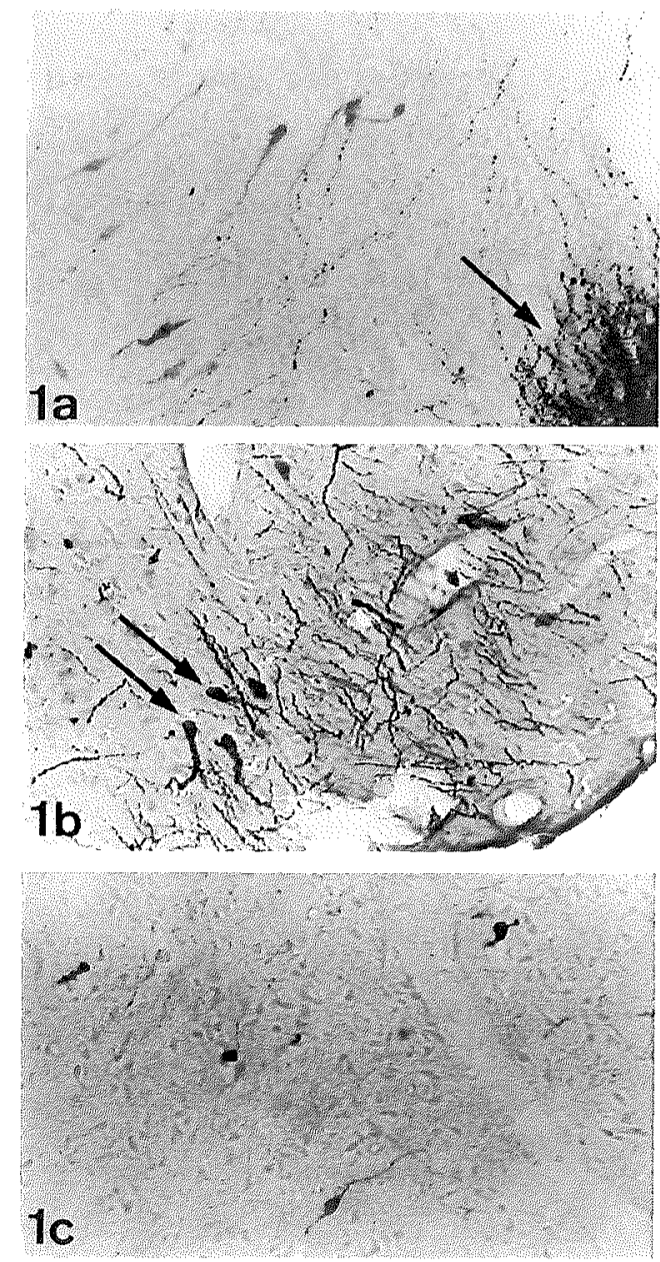

Fig. 1a LHRH immunoreactive neuronal perikarya distributed in a region dorsolateral to the OVLT. Large numbers of immunoreactive fibers are also found in the OVLT (arrow). $\times 185$

Fig. 1b LHRH immunoreactive neuronal perikarya (arrows) in the lateral part of the pars suprachiasmatica of the POA. There are also many immunoreactive fibers. $\times 185$

Fig. 1c Sparsely distributed LHRH neuronal perikarya in the rostral part of the anterior hypothalamic nucleus. $\times 155$

ular nucleus and the arcuate nucleus existed in the midbrain, LHRH immunoreactive somata were never detected.

\section{Distribution of Immunoreactive Fibers and Terminals}

Immunoreactive LHRH fibers were seen as thin strands or varicosities strongly stained brown. 

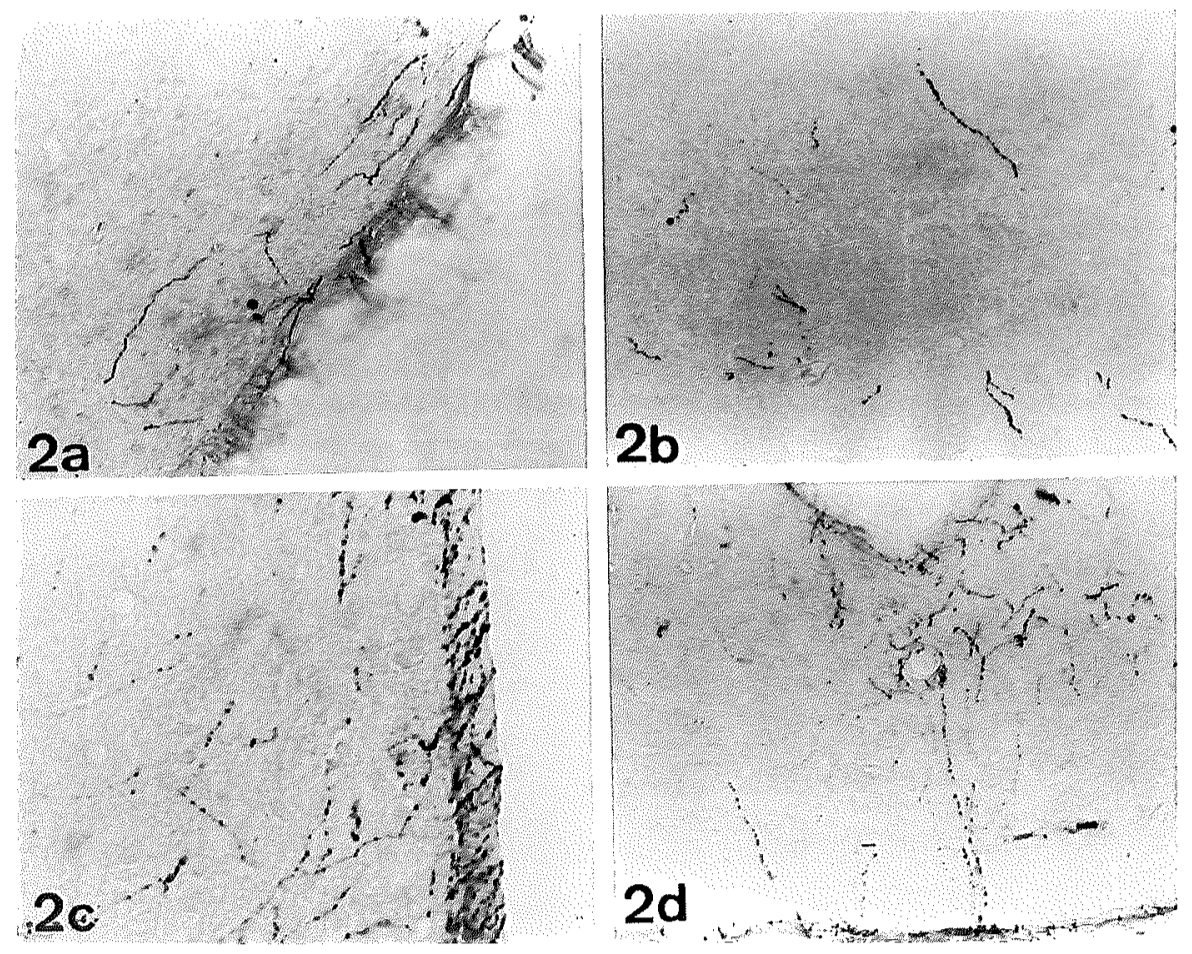

Fig. 2a Small numbers of LHRH fibers in the mediobasal part of the olfactory bulb. $\quad \times 120$ Fig. $2 b$ Small numbers of varicose LHRH fibers in the medial septal nucleus. $\times 120$

Fig. 2c A large number of LHRH nerve fibers observed in close contact with the surface of the third ventricle at the level of the POA. $\times 270$

Fig. 2d LHRH immunoreactive fibers extending perpendicularly through the optic chiasma. $\times 120$

In the most rostral region they were found in small numbers in the mediobasal and laterobasal part of the olfactory bulb (Fig. 2a). They were less abundant in the laterobasal part than in the mediobasal part. In the more caudal portion, a small number were also found in the vicinity of the diagonal bundle of Broca. At the level of the OVLT relatively large numbers of immunoreactive fibers were detected around the OVLT, particularly in its dorsolateral part. At the same frontal level immunoreactive fibers extending to the dorsoventral direction were detected in the medial and lateral septal nuclei (Fig. 2b). At a more caudal level considerable numbers of immunoreactive fibers extended from the basolateral part of the POA to the optic chiasma. We also found some fibers with strong immunoreactivity running perpendicularly along the third ventricle. Many of their tips seemed to be in contact with the ependymal surface of the third ventricle (Fig. 2c). Immunoreactive fibers extending perpendicularly through the optic chiasma were observed in the base of the brain (Fig. 2d), apparently extending to a caudal direction. At the level between the anterior hypothalamic nucleus and the paraventricular nucleus (PVN), large numbers of immunoreactive fibers were found extending to a lateromedial direction in the mediobasal hypothalamus. Another group of immunoreactive fibers ran to a dorsoventral direction along the third ventricle. Imunoreactive fibers were sparsely distributed in the dorsal part of the mamillary body.

Besides the olfactory bulb and the diagonal bundle of Broca, LHRH immunoreactive fibers and terminals were detected in the extrahypothalamic regions, i.e. the piriform cortex (Fig. $3 a)$, the cingulate gyrus, the medial and basal amygdaloid nuclei, the subfornical organ, the mediodorsal thalamic nucleus, the habenular nucleus, the central grey of the midbrain, and the lateral part of the interpeduncular nucleus (Fig. 3b). Relatively large numbers of immuno- 

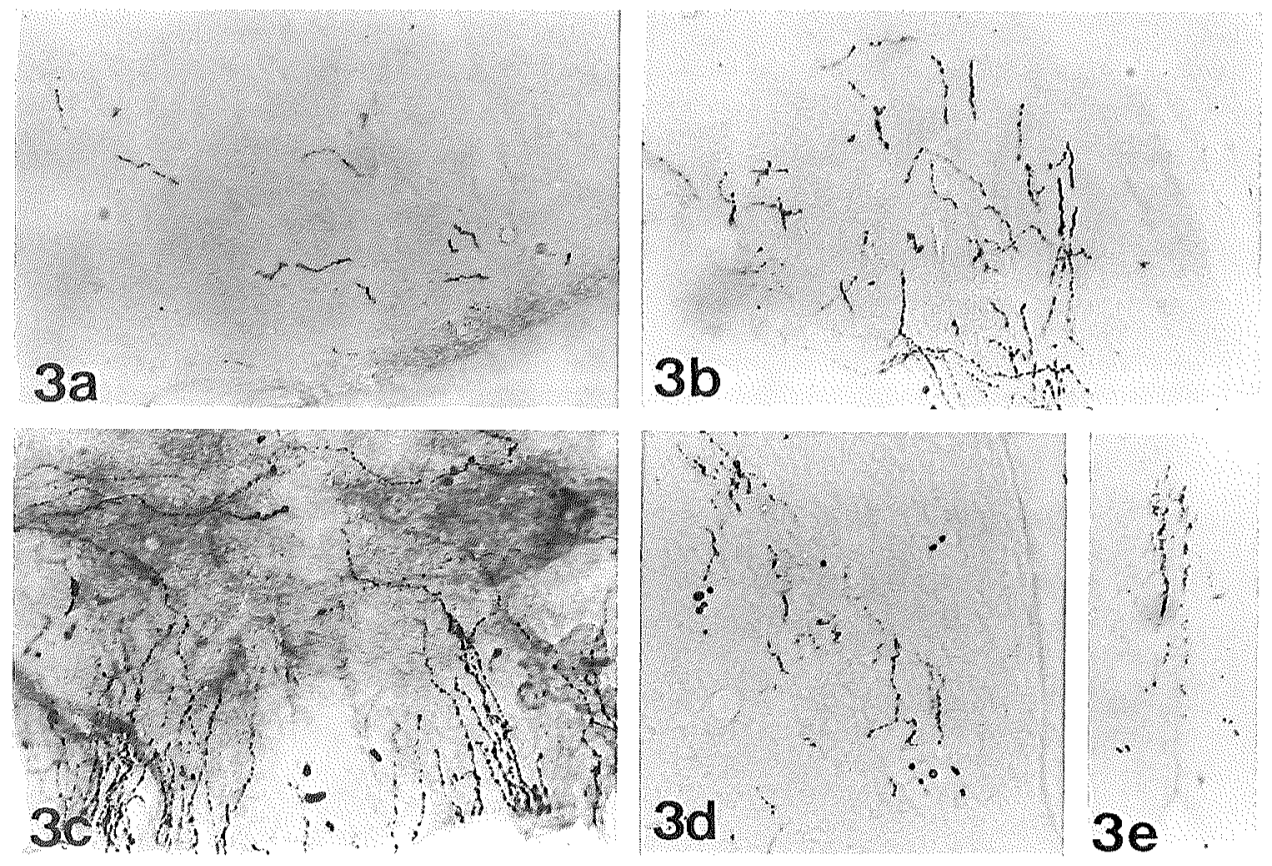

Fig. 3a A small number of LHRH fibers in the piriform cortex. $\times 130$

Fig. 3b LHRH fibers distributed in the lateral part of the interpeduncular nucleus. $\times 130$

Fig. 3c Numerous LHRH immunoreactive fibers with varicosities distributed in the subfornical organ. $\times 260$

Fig. 3, d and e Immunoreactive LHRH fibers in the medial habenular nucleus (d) and the central grey of the midbrain (e). $\mathrm{d}: \times 130, \mathrm{e}: \times 65$

reactive varicosities were distributed in the subfornical organ and a considerable proportion of them seemed to terminate around the capillaries of the ventricle (Fig. 3c). Immunoreactive fibers were seen scattered in the medial habenular nucleus (Fig. 3d) and the commissura habenularum. Immunoreactive fibers were also detected in the central grey of the midbrain (Fig. 3e). Some of them were situated very close to the aqueduct cerebri. In the other parts, they were observed as brown immunoreactive strands running in various directions.

A dense accumulation of LHRH terminals was found in the lateral part of the external layer of the ME (Fig. 4a) and in the OVLT (Fig. 4b), both of which are generally accepted to be main terminal areas. The location of LHRH somata and the distribution of their fibers and terminals revealed in this study are shown schematically in Fig. 5.

\section{DISCUSSION}

Our immunohistochemical investigation has revealed the presence of LHRH neuronal somata and the widespread distribution of their processes by using the same anti-sera used in our previous study, in which we had failed to detect neuronal somata. The technical revisions in procedures, including changes in fixation and adjustment of immunoreaction periods, appear to have caused a significant improvement (5).

Nearly all the immunohistochemical investigations of LHRH in the rodent brain have been performed in combination with experimental interference, such as colchicine injection or administration of nembutal or reserpine $(4,23$, 27 ), to demonstrate their somata. In this study, we were able to detect somata under completely physiological conditions without any such treatments $(5,17)$. Neuronal somata are widely spread in a rostrocaudal direction from the diagonal bundle of Broca to the anterior hypothalamic nucleus, and their main location is considered to be the basolateral part of the POA and the POSA. We were unable to detect immunoreactive somata in the arcuate nucleus in agreement with Bennett-Clarke and Joseph 

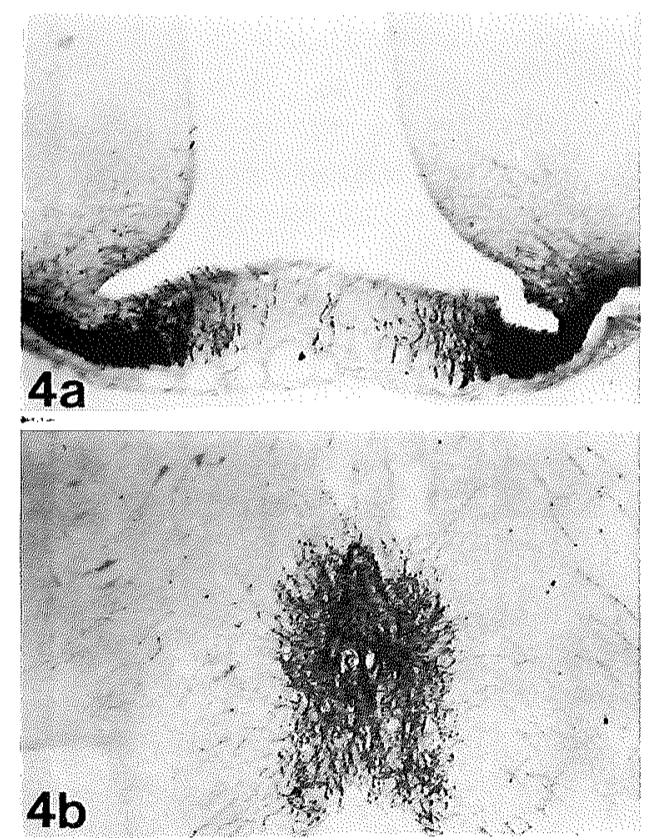

Fig. 4a Large numbers of LHRH nerve terminals in the lateral part of the external layer of the ME. A few immunoreactive fibers are also seen in the medial part. $\times 60$

Fig. $4 \mathrm{~b}$ Accumulation of LHRH immunoreactive fibers and terminals in the OVLT. $\times 60$

(5), despite its detection by some investigator's $(22,28)$. The neuronal somata in the arcuate nucleus may be other types of peptidergic neurons, such as ACTH (6). However, the extent of immunoreactivity of LHRH in somata was clearly less than that in neuronal processes and terminals. Therefore, we support the opinion that the intracellular accumulation of LHRH in the rodent brain is insufficient to be detectable by ordinary methods of immunohistochemistry using Bouin's fixative (2).

Extrahypothalamic LHRH fibers and terminals have been demonstrated mainly in the guinea pig $(3,25)$ and golden hamster (13). However, a few papers partially demonstrating them in the rat have been available $(5,19)$. In study we found that the distribution of extrahypothalamic LHRH fibers and terminals have a close similarity to that of the guinea pig (25), and the golden hamster (13). In the rat, we were able to identify the basal rhinencephalic LHRH pathway arising from somata of the rostral groups, including the diagonal bundle of Broca, and projecting into the olfactory bulb; and the septothalamic and septohabenular pathway, which runs probably through the striamedullaries, as shown in the golden hamster (13) and other species (2). Numerous immunoreactive fibers and terminals were found in the subfornical organ, from which some seem to be released into the ventricle. We found immunoreactive fibers and terminals in the central grey of the midbrain and in the interpeduncular nucleus. In the central grey they are located adjacent to the ependyma surrounding the mesencephalic aqueductus as demonstrated by Liposits and Sétáló (19). They seem to travel in the fasciculus retroflexus. A few LHRH terminals were also detected in the retromammillary area and in the mammillary body. Parasagittal sections indicate that their pathway may be through the periventricular region in the diencephalon.

In a previous paper dealing with the pathways of $\mathrm{LHRH}$ neurons projecting to the ME, we described two pathways (the main baso-lateral pathway and the accessory descending pathway), and suggested that the location of their original neuronal somata was in the ventral part of the medial part of POA. These results are essentially in agreement with those of Sétáló et al. (23), which have been confirmed also by other investigations $(5,7,13,20)$. This study also confirms our previous finding that LHRH neurons located in the lateral part of the POA and in the lateral part of the anterior hypothalamic nucleus project to the ME, and that the third pathway running along the base of the diencephalon also projects to the ME. This seems to correspond to the pathway along the ventral surface of the brain under the optic chiasma demonstrated by Hoffman and Gibbs (7).

Many LHRH terminals in the OVLT seem to arise from neuronal somata located in the vicinity, since numerous immunoreactive neuronal somata were found around the OVLT.

A considerable number of LHRH terminals are also distributed around perivascular spaces in the subfornical organ, particularly in its lateral part, as mentioned by Krisch and Leonhardt (18), though they are also distributed in its medial part. They are probably released into the capillaries.

Numerous LHRH immunoreactive processes were in contact with the surface of the third ventricle in the diencephalon and midbrain, particularly at the anterior hypothalamic nucleus and the arcuate nucleus level. These observations support the view that LHRH is released into the third ventricle $(5,16)$. To obtain 

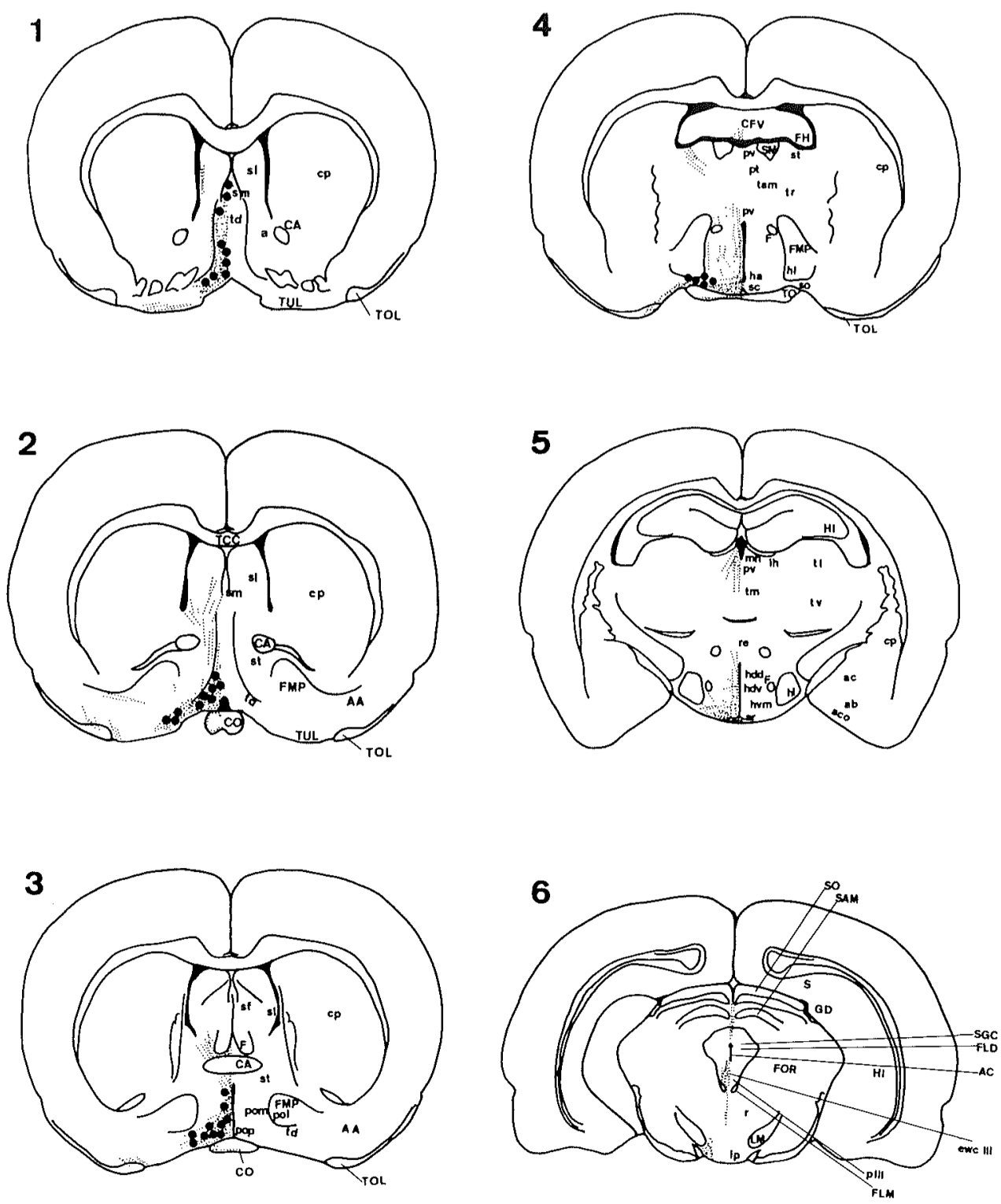

Fig. 5 Diagram showing the location of LHRH neuronal somata, fibers, and terminals. Large dots indicate neuronal somata and small dots indicate fibers and terminals. Their number corresponds to the distributional density of the somata, fibers and terminals.

Abbreviations: AA: Area amygdala anterior. AC: Aqueductus cerebri (sylvii). CA: Commissura anterior. CFV: Commissura fornicis ventralis (Commissura hippocampi ventralis). CO: Chiasma opticum. F: Columna fornicis. FH: Fimbria hippocampi. FLD: Fasciculus longitudinalis dorsalis. FMP: Fasciculus medialis prosencephali. FOR: Formatio reticularis. GD: Gyrus dentatus. HI: Hippocampus. LM: Lemmiscus medialis. SAM: Stratum album mediale colliculi superioris. SGC: Substantia grisea centralis. SM: Stria medullaris thalami. SO: Stratum opticum colliculi superioris. ST: Stria terminalis. TCC: Truncus corporis callosi. TO: Tractus opticus. TOL: Tractus olfactoris lateralis. TUL: Tuberculum olfactorium, pars corticalis. a: Nucleus accumbens. abm: Nucleus amygdaloideus basalis, pars medialis, aco: Nucleus amygdaloideus corticalis. am: Nucleus amygdaloideus medialis. ar: Nucleus arcuatus. cp: Nucleus caudatus putamine. ewc III: Nucleus Edinger-Westphal n. oculomotorii, pars caudalis, ha: Nucleus anterior (hypothalami). hl: Nucleus lateralis (hypothalami). hpv: Nucleus periventricularis (hypothalami). hvm: Nucleus ventromedialis (hypothalami). ip: Nucleus interpeduncularis. pol: Nucleus preopticus lateralis. pop: Nucleus preopticus periventricularis. pt: Nucleus paratenalis. pv: Nucleus premamillaris ventralis. p: 
further information on the precise nature and function of LHRH, electron microscopic studies will be required.

This work was supported in part by grants (Nos. 248093, 321426 and 57370002) from the Ministry of Education, Science and Culture, Japan.

Received for publication 9 January 1983; and in revised form 7 February 1983

\section{REFERENCES}

1. Baker B. L., Dermody W. C. and Reel J. R. (1974) Localization of luteinizing hormonereleasing hormone in the mammalian hypothalamus. Amer. J. Anat. 139, 129-134

2. BARRY J. (1979) Immunohistochemistry of luteinizing hormone-releasing hormone-producing neurons of the vertebrates. Int. Rev. Cytol. 60, 179-221

3. Barry J, and DuboIs M. P. (1974) Immunofiuorescence study of the preoptico-infundibular LH-RH neurosecretory pathway of the guinea pig during the estrous cycle. Neuroendocrinology 15, 200-208

4. Barry J., Dubois M. P. and Poulain P. (1973) LRF producing cells of the mammalian hypothalamus. A fluorescent antibody study, $Z$. Zellforsch. 146, 351-366

5. Bennett-Clarke C, and Joseph S. A. (1982) Immunocytochemical distribution of LHRH neurons and processes in the rat: Hypothalamic and extrahypothalamic locations. Cell Tissue Res. 221, 493-504

6. Clayton C. J. and Hoffman G. E. (1979) Immunocytochemical evidence for anti-LHRH and anti-ACTH activity in the " $F$ " antiserum (1). Amer. J. Anat. 155, 139-145

7. Hoffman G. E. and GibBs F. P. (1982) LHRH pathways in rat brain: 'Deafferentation' spares a sub-chiasmatic LHRH projection to the median eminence. Neuroscience 7, 1979-1993

8. Hoffman G. E., Melnyk V., Hayes T., BennettClarke C. and Fowler E. (1978) Immunocytology of LHRH neurons. In Brain-Endocrine Interaction. III. Neural hormones and reproduction (ed. Scott D. E., Kozlowski G. P. and WeINDL A.) Karger, Basel, pp. 67-82

9. Ibata Y. and Watanabe K. (1977) A morphological survey of the median eminence: Fluorescence histochemistry, electron microscopy and immunohistochemistry. Arch. histol. japon. 40, Suppl., 303-315

10. Ibata Y., Watanabe K., Kimura H., Sano Y.,
Sin S., Hashimura E. and Imagawa K. (1978) Distribution of LH-RH nerve endings in the median eminence of proestrus female rats: Fluorescence and peroxidase anti-peroxidase (PAP) immunohistochemistry. Endocrinol. Japon. 25, 141-148

11. Ibata Y., Watanabe K., Kinoshita H., Kubo S., Sano Y., Sin S., Hashimura E. and Imagawa K. (1979) The location of LH-RH neurons in the rat hypothalamus and their pathways to the median eminence. Experimental immunohistochemistry and radioimmunoassay. Cell Tissue Res. 198, 381-395

12. Jennes L., and StumpF W. E. (1980) LHRHsystems in the brain of the golden hamster. Cell Tissue Res. 209, 239-256

13. Kawano H. and Daikoku S. (1981) Immunohistochemical demonstration of LHRH neurons and their pathways in the rat hypothalamus. Neuroendocrinology' 32, 179-186

14. King J. C., Parsons J. A., Erlandsen S. L. and Williams T. H. (1974) Luteinizing hormonereleasing hormone (LH-RH) pathway of the rat hypothalamus revealed by the unlabeled antibody peroxidase-antiperoxidase method. Cell Tissue Res, 153, 211-217

15. Knigge K. M., Joseph S. A., Hoffman G. E., Arimura A. and Sternberger L. (1978) Organization of LRF- and SRIF-neurons in the endocrine hypothalamus. In The hypothalamis (ed. Reichlin S., Baldessarini R. J. and MaRTIN J. B.) Raven Press, New York, pp. 49-67

16. Knigge K. M., Joseph S. A., Sladek J. R., Notter M. F., Morris M., Sundberg D. K., Holzwarth M. A., Hoffman G. E. and O'BRIEN L. (1976) Uptake and transport activity of the median eminence of the hypothalamus. Int. Rev. Cytol. 45, 383-408

17. KrIsCH B. (1980) Two types of luliberin-immunoreactive perikarya in the preoptic area of the rat. Cell Tissue Res. 212, 443-455

18. KRISCH B. and Leonhardt H. (1980) Luliberin and somatostatin fiberterminals in the subfornical organ of the rat. Cell Tissue Res. 210, $33-45$

19. Liposits Zs. and SÉTÁLÓ G. (1980) Descending luteinizing hormone-releasing hormone (LH$\mathrm{RH})$ nerve fibers to the midbrain of the rat. Neurosci. Lett. 20, 1-4

20. Merchenthaler I., Kovács G., Lovász G. and SÉTÁló G. (1980) The preoptico-infundibular LH-RH tract of the rat. Brain Res. 198, 63-74

21. Merchenthaler I., Lengvári I., Horváth J. and SÉTÁLÓ G. (1980) Immunohistochemical study of the LHRH-synthesizing neuron system

Nucleus principalis $n$. oculomotorii. $\mathrm{r}:$ Nucleus ruber. re: Nucleus rentiens, rh: Nucleus rhomboidens. sc: Nucleus suprachiasmaticus. sf: Nucleus septalis fimbrialis. sl: Nucleus septi lateralis. sm: Nucleus septi medialis, so: Nucleus supraopticus. st: Nucleus interstitialis striae terminalis, tam: Nucleus anterior medialis thalami. tav: Nuclens anterior ventralis thalami. td: Nucleus tractus diagonalis (Broca). tl: Nucleus lateralis thalami. $\mathrm{tmm}$ : Nucleus medialis thalami, pars medialis, $\operatorname{tr}:$ Nucleus reticularis thalami. tv: Nucleas ventralis thalami 
of aged female rats. Cell Tissue Res. 209, 499503

22. NAIK D. V. (1975) Immunoreactive LH-RH neurons in the hypothalamus identified by light and fluorescent microscopy. Cell Tissue Res. 157, 423-436

23. Sétáló G., Vigh S., Schally A. V., Arimura A. and Flerkó B. (1976) Immunohistological study of the origin of LH-RH-containing nerve fibers of the rat hypothalamus. Brain Res. 103, 597602

24. Silverman A. J. (1976) Distribution of luteinizing hormone-releasing hormone (LHRH) in the guinea pig brain. Endocrinology 99, 30-41

25. Silverman A. J. and Krey L. C. (1978) The luteinizing hormone-releasing hormone (LH-
RH) neuronal networks of the guinea pig brain. I. Intra- and extra-hypothalamic projections. Brain Res. 157, 233-246

26. Sternberger L. A. and Hoffman G. E. (1978) Immunocytology of luteinizing hormonereleasing hormone. Neuroendocrinology $\mathbf{2 5}$, $111-128$

27. Vigh S., Sétáló G., Schally A. V., Arimura A. and FLERKO B. (1978) LH-RH-containing nerve fibers in the brain of rats treated with sulpiride or reserpine. Brain Res. 152, 401-405

28. Zimmerman E. A., Hsu K. C., Ferin M. and Kozlowski G. P. (1974) Localization of gonadotropin-releasing hormone $(\mathrm{Gn}-\mathrm{RH})$ in the hypothalamus of the mouse by immunoperoxidase technique. Endocrinology 95, 1-8 